\title{
Quenching of Star-formation Activity of High-redshift Galaxies in Clusters and Field
}

\author{
Seong-Kook Lee ${ }^{1}$, Myungshin Im ${ }^{1}$, Jae-Woo Kim ${ }^{1}$, Jennifer Lotz ${ }^{2}$, \\ Conor McPartland ${ }^{3}$, Michael Peth ${ }^{4}$ and Anton Koekemoer ${ }^{2}$ \\ ${ }^{1}$ CEOU/Department of Physics and Astronomy, Seoul National University, Korea, email: \\ sklee@astro.snu.ac.kr, ${ }^{2}$ STScI, Baltimore, USA, ${ }^{3}$ IfA, University of Hawaii, Honolulu, USA, \\ ${ }^{4}$ Department of Physics and Astronomy, Johns Hopkins University, Baltimore, USA
}

\begin{abstract}
At local, galaxy properties are well known to be clearly different in different environments. However, it is still an open question how this environment-dependent trend has been shaped. We present the results of our investigation about the evolution of star-formation properties of galaxies over a wide redshift range, from $z \sim 2$ to $z \sim 0.5$, focusing its dependence on their stellar mass and environment (Lee et al. 2015). In the UKIDSS/UDS region, covering $\sim 2800$ square arcmin, we estimated photometric redshifts and stellar population properties, such as stellar masses and star-formation rates, using the deep optical and near-infrared data available in this field. Then, we identified galaxy cluster candidates within the given redshift range. Through the analysis and comparison of star-formation (SF) properties of galaxies in clusters and in field, we found interesting results regarding the evolution of SF properties of galaxies: (1) regardless of redshifts, stellar mass is a key parameter controlling quenching of star formation in galaxies; (2) At $z<1$, environmental effects become important at quenching star formation regardless of stellar mass of galaxies; and (3) However, the result of the environmental quenching is prominent only for low mass galaxies $\left(\mathrm{M}_{*}<10^{10} \mathrm{M}_{\odot}\right)$ since the star formation in most of high mass galaxies are already quenched at $z>1$.
\end{abstract}

Keywords. galaxies: clusters: general, galaxies: high-redshift, galaxies: evolution
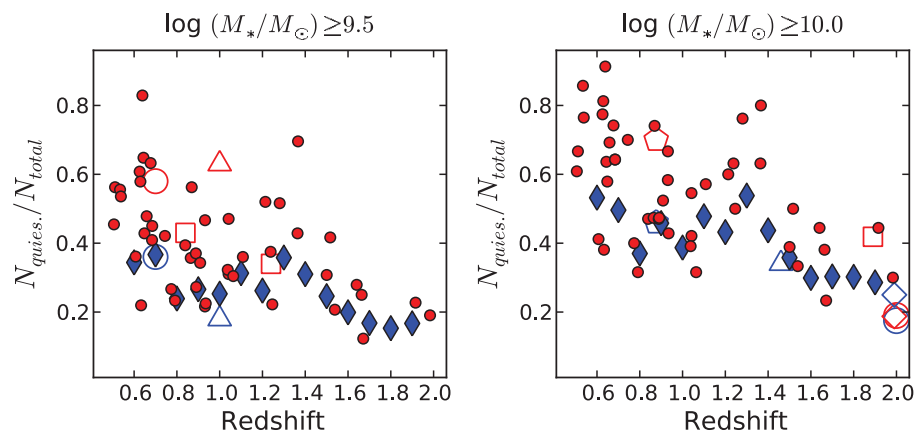

Figure 1. The evolution of quiescent galaxy fraction with different stellar-mass limits (Left: log $\left(\mathrm{M}_{*} / \mathrm{M}_{\odot}\right) \geqslant 9.5$, Right: $\left.\log \left(\mathrm{M}_{*} / \mathrm{M}_{\odot}\right) \geqslant 10.0\right)$. The red filled circles show the individual clusters and the blue filled diamonds are the values of field galaxies at each redshift bin $(\Delta z=0.1)$. The difference of quiescent fraction with different stellar mass limits is greater than the difference between cluster and field galaxies with given stellar mass limit. Environmental dependence of quiescent galaxy fraction becomes significant at $z<1$.

\section{Reference}

Lee, S.-K., Im, M., Kim, J.-W., Lotz, J., McPartland, C., Peth, M., \& Koekemoer, A. 2015, ApJ, 810, 90 